\title{
VLBI Observations of the Gravitational Lens B1422+231
}

\author{
A. R. Patnaik \& R. W. Porcas \\ Max-Planck-Institut für Radioastronomie, Bonn, Germany
}

Abstract. We present results from VLBI observations of the gravitational lens B1422+231. All the four images of this system have been detected and appear unresolved at $18 \mathrm{~cm}$. Images $A$ and $B$ show elongated structures at $6 \mathrm{~cm}$ with 1 mas resolution. With a resolution of 0.75 mas at $2 \mathrm{~cm}$, three images are elongated with axial ratios of about 10:1 for $A$ and $B$, and $2: 1$ for $C$.

\section{Introduction}

The gravitational lens system B1422+231 consists of 4 images with maximum image separation of 1.3 arcsec (Patnaik et al. 1992). It is associated with a $15.5 \mathrm{mag}$ quasar at $\mathrm{z}=3.62$. The flux ratios of images $\mathrm{A}, \mathrm{C}$ and $\mathrm{D}$ with respect to $\mathrm{B}$ are $1.0,0.5,0.02$. The images have similar fractional polarization as well as similar PA of polarization. Recent HST observations detect all 4 images as well as the lensing galaxy and the spectra of the images are virtually identical (Impey et al. 1996).

\section{Observations and Results}

Global MK3 observations at $18 \mathrm{~cm}$ and $6 \mathrm{~cm}$ were made in $1991 / 2 ; 6 \mathrm{~cm}$ maps are given in Patnaik \& Porcas (1996). New $2 \mathrm{~cm}$ observations were made with the VLBA on 1995 May 26 . A total of $64 \mathrm{MHz}$ bandwidth was used in a single polarization. The data were analyzed using the NRAO AIPS software package.

It is rather remarkable that the images $A$ and $B$ are elongated by about 10:1 while image $\mathrm{C}$ is elongated by $2: 1$. The deconvolved sizes are $\mathrm{A}: 2.35 \times 0.27$ mas at $57^{\circ} ; \mathrm{B}: 2.90 \times 0.26$ mas at $45^{\circ} ; \mathrm{C}: 1.30 \times 0.56$ mas at $27^{\circ}$. Of particular interest is the PA of these elongations as they form an arc, which is expected from an elliptical lens. Thus we believe that most of the elongation is due to "tangential stretching" rather than internal structure of the radio source.

The flux ratios (Table 1) derived from these $2 \mathrm{~cm}$ observations compare well with results from other radio frequencies. There is significant difference in the flux ratio of image A at IR and optical bands with respect to radio bands. It is possible that this image is affected by microlensing which can affect the optical and IR structures much more than the radio.

Acknowledgments. The National Radio Astronomy Observatory is a facility of the National Science Foundation, operated under a cooperative agreement by Associated Universities, Inc.

\section{References}

Impey, C. D. et al. 1996. $A p J, 462, \mathrm{~L} 53-57$.

Lawrence, C. R. et al. 1992. MNRAS, 259, 5-7p.

Patnaik, A. R. et al. 1992. MNRAS, 259, 1-4p.

Patnaik, A. R., \& Porcas, R. W. 1996. in Astrophysical Applications of Gravitational Lensing: IAU Symp. 173 eds. C. S. Kochanek \& J. N. Hewitt (Dordrecht: Kluwer), 305-310. 


\begin{tabular}{lllllll}
\hline \hline & & & & & & \\
Component & $18 \mathrm{~cm}$ & $6 \mathrm{~cm}$ & $2 \mathrm{~cm}$ & IR & Opt. Cont. & Ly $\alpha$ \\
\hline & & & & & & \\
A & 1.02 & 0.96 & 1.00 & 0.78 & 0.78 & 0.90 \\
B & 1.00 & 1.00 & 1.00 & 1.00 & 1.00 & 1.00 \\
C & 0.59 & 0.47 & 0.49 & 0.49 & 0.50 & 0.49 \\
D & 0.023 & 0.08 & 0.03 & 0.06 & 0.025 & 0.027 \\
\hline \hline
\end{tabular}

Table 1. Flux ratios of the images with respect to image $B$ at several wavelengths. The radio flux ratios are from global VLBI and VLBA observations. Optical flux ratios are from Impey et al. (1996), IR values are from Lawrence et al. (1992).

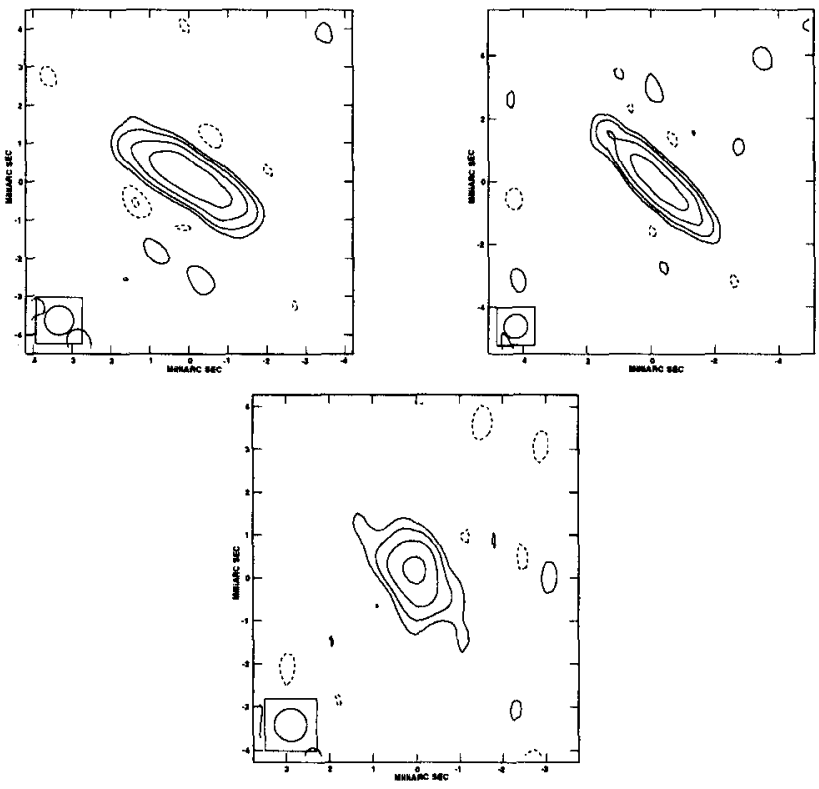

Figure 1. VLBA maps of B1422+231 A (top left), B (top right) and C (bottom) at $15 \mathrm{GHz}$ with a resolution of 0.75 mas. Image $D$ is detected only in natural weighted maps. The restoring beam is plotted at the lower left hand corner of each map. Contour levels are in $1.5 \mathrm{mJy} /$ beam $\times-2,-1,1,2,4,8,16,32$. 